\title{
MARK-III VLBI ASTROMETRY OF PULSARS
}

\author{
DAYTON L. JONES
}

Jet Propulsion Laboratory, Mail Code 238-700, California Institute of Technology, Pasadena, CA. 91109

RACHEL J. DEWEY

Jet Propulsion Laboratory, Mail Code 238-700, California Institute of Technology, Pasadena, CA. 91109

CARL R. GWINN

Dept. of Physics, University of California, Santa Barbara, CA. 93106

MICHAEL M. DAVIS

National Astronomy and Ionosphere Center, Arecibo Observatory, P. O. Box 995, Arecibo, P.R. 00613

\begin{abstract}
Differential 18-cm VLBI measurements of the position of PSR $1937+214$ with respect to the quasar $1923+210$ have been made to improve our knowledge of the offset between the planetary ephemeris reference frame and the extragalactic radio reference frame. Our preliminary frame-tie result (VLBI - Timing position) is $+21 \pm 2$ milli-arcseconds in right ascension and $+14 \pm 9$ milli-arcseconds in declination. We describe the data analysis methods used and compare our results with other radio interferometric position measurements of PSR $1937+214$. Our results illustrate the value of combining the large collecting area of the Arecibo telescope with the Mk-III VLBI recording system.
\end{abstract}

\section{INTRODUCTION}

Astrometric VLBI observations of pulsars with milli-arcsecond (mas) positions from pulse arrival time measurements can be used to relate the reference frames defined by VLBI and planetary ephemerides. The VLBI reference frame is defined by extensive observations of compact extragalactic radio sources by NASA, JPL, and the National Geodetic Service. Due to the great distance of extragalactic sources, the VLBI reference frame is believed to be very nearly inertial. Pulsar timing measurements yield pulsar positions with respect to the Earth's orbit. Thus, observations of the same pulsar(s) with both techniques can determine the offset between the two reference frames. Knowledge of the offset between the planetary ephemeris reference frame and the extragalactic radio reference frame is important for 1 ) navigation of 
interplanetary spacecraft, 2) measurement of rotation between the frames via long-term monitoring, and 3) referencing studies of relativistic solar system dynamics to the extragalactic frame.

The VLBI observations require the maximum possible sensitivity, because the most accurate timing positions are those for pulsars with millisecond periods (which tend to have much smaller intrinsic fluctuations in their pulse arrival times than slower pulsars) and these pulsars have very low flux densities at $\mathrm{GHz}$ frequencies where accurate VLBI astrometry is possible. The best candidate is PSR $1937+214$, which has no timing noise at the microsecond level and consequently a position (in the ephemeris frame) with accuracy of less than a milli-arcsecond (Backer et al. 1985; Davis et al. 1985; Rawley et al. 1988). There are other millisecond pulsars with very low timing noise, but PSR $1937+214$ is by far the strongest at high radio frequencies.

\section{OBSERVATIONS}

The VLBI observations of PSR 1937+214 reported here were made in March 1990 using Arecibo, the full VLA, and the 70-m DSN antennas at Goldstone, California, and Madrid, Spain. We observed at a wavelength of $18 \mathrm{~cm}$, using the $\mathrm{Mk}$-III system in mode $\mathrm{C}$ with fourteen $2-\mathrm{MHz}$ channels spread over a total bandwidth of $40 \mathrm{MHz}$. The total bandwidth was limited to $40 \mathrm{MHz}$ by the line feed used at Arecibo. The data were cross-correlated at Haystack, using a new fast pulsar gating system developed by R. Cappallo. Fringe-fitted data were transported to JPL for analysis with MODEST, a new version of the standard MASTERFIT astrometry/geodesy program developed by 0 . Sovers.

We observed PSR $1937+214$ and an extragalactic reference source $(1923+210=$ OV239.7 $)$ for several hours, alternating between sources with a cycle time of 8-10 minutes. The reference source is about $3^{\circ}$ away from the pulsar, and is observed regularly by JPL and the NASA Crustal Dynamics Program.

\section{ANALYSIS}

For $1923+210$ we adopt the following J2000 position from the JPL reference source catalog:

$$
\alpha=19^{\mathrm{h}} 25^{\mathrm{m}} 59: 60533 \pm 0: 00003, \quad \delta=+21^{\circ} 06^{\prime} 26^{\prime} \cdot 1626 \pm 0^{\prime} .0007
$$

The initial fits for station clocks, clock rates, ionosphere and troposphere delays, using data from the reference source only, produced large systematic residuals on baselines to Arecibo. We believe that this was due to an inaccurate position for Arecibo. Varying the position of Arecibo to minimize the residuals resulted in the following geocentric coordinates for the center of curvature of the spherical reflector:

$$
\mathrm{X}=2390490 . \quad \mathrm{Y}=-5564731 . \quad \mathrm{Z}=1994727 .
$$


This differs from the Arecibo position used at the correlator by $\approx 8 \mathrm{~m}$, largely in $\mathrm{Z}$. Using our new position, the rms scatter of the post-fit residuals was reduced to 0.2-0.6 ns in group delay and 1-2 ps/s in delay rate.

Solving for the pulsar position with respect to the calibrator position resulted in an offset (VLBI - timing) of

$$
\Delta \mathrm{RA}=+21.1 \pm 1.0 \text { mas, } \quad \Delta \mathrm{DEC}=+10.9 \pm 6.0 \text { mas }
$$

from the published DE200 (J2000) timing position (Backer et al. 1985). The formal errors are from MODEST's multi-parameter least-squares fit, and do not include systematic errors. In addition to the pulsar position, MODEST simultaneously fit for station clock offsets, clock rates, tropospheres, ionospheres, and the position of Arecibo.

We then corrected for the pulsar's proper motion during the 7.3 years between the timing position epoch and our VLBI epoch, assuming proper motions of $-0.04 \mathrm{mas} /$ year in right ascension and -0.42 mas/year in declination (M. Ryba, private communication). The resulting VLBI - timing position offset is

$$
\Delta \mathrm{RA}=+21.4 \pm 1.1 \text { mas, } \quad \Delta \mathrm{DEC}=+14.0 \pm 6.3 \operatorname{mas}
$$

where the errors now include the formal VLBI position errors, published timing position errors, and an assumed error of up to $50 \%$ in the proper motion values. Systematic errors could increase the final offset errors, and are discussed below.

\section{RESULTS}

Our preliminary results are summarized in Table 1, which lists the DE200 timing position, the timing position corrected for proper motion, and our VLBI position.

TABLE I J2000 Positions for PSR 1937+214

\begin{tabular}{lcc}
\hline Source & Right Ascension & Declination \\
\hline Timing & $19^{\mathrm{h}} 39^{\mathrm{m}} 38: 56025 \pm 0.00003$ & $+21^{\circ} 34^{\prime} 59^{\prime} .145 \pm 0.001$ \\
Timing + PM & $19^{\mathrm{h}} 39^{\mathrm{m}} 38^{s}: 56023 \pm 0.00004$ & $+21^{\circ} 34^{\prime} 59^{\prime} .142 \pm 0.002$ \\
VLBI & $19^{\mathrm{h}} 39^{\mathrm{m}} 38^{s} 56176 \pm 0: 00008$ & $+21^{\circ} 34^{\prime} 59^{\prime} .156 \pm 0.006$ \\
\hline
\end{tabular}

Systematic errors are difficult to estimate accurately, but since this is a differential experiment we are only concerned with systematic errors due to variations in the ionosphere, troposphere, etc., over the few degrees between the two sources, rather then errors in the absolute values of these parameters. Based on the results of multiple MODEST fits using different starting values, different subsets of our six baselines, and solving for different sets of parameters, we believe that the systematic error in right ascension is 
no larger than 1.5 mas. The variation in declination is larger, suggesting that systematic errors may be limiting our accuracy in this coordinate to 7-8 mas. Ways to reduce the declination error are currently being studied.

Combining the random measurement errors with our present estimate of the systematic errors gives the following position difference (VLBI - timing):

$$
\Delta \mathrm{RA}=+21 . \pm 2 \text { mas, } \quad \Delta \mathrm{DEC}=+14 . \pm 9 \text { mas }
$$

This frame-tie determination is consistent in right ascension (within the rss combined errors) with the Bartel et al. (1990) result. However, our declination value differs by 30 mas from that of Bartel et al., which is just over three times the rss combined errors. Our VLBI position for PSR 1937+214 is nearly identical to the VLA position determined by Backer et al. (1985), but this is coincidental given the 40-50 mas errors on the VLA measurement.

A complete determination of the offset between two reference frames requires a minimum of two measurements at different locations on the sky. We hope to use this technique on additional millisecond pulsars, but this may require further improvements in the sensitivity of VLBI systems. It will also be useful to repeat this experiment on PSR $1937+214$ after several years to determine if there is a rotation between the planetary and extragalactic frames at the mas/year level.

\section{ACKNOWLEDGEMENTS}

We are grateful to the VLBI network for observing time, and to R. Cappallo for developing the fast correlator gating system at Haystack, R. Phillips and A. Rogers for assistance with the fringe-fitting software at Haystack, and 0 . Sovers for many useful discussions about MODEST. R. Linfield initiated JPL's effort to improve the planetary-extragalactic reference frame tie with VLBI astrometry of millisecond pulsars, and has provided helpful guidance to this project. Portions of this research were carried out at the Jet Propulsion Laboratory, California Institute of Technology, under contract with the National Aeronautics and Space Administration.

\section{REFERENCES}

Backer, D. C., et al. 1985, A. J., 90, 2275.

Bartel, N., et al. 1985, A. J., 90, 318.

Bartel, N., et al. 1990, in Workshop on the Impact of Pulsar Timing on Relativity and Cosmology (Berkeley, June 1990), p. X1.

Davis, M. M., et al. 1985, Nature, 315, 547.

Rawley, L. A., Taylor, J. H., and Davis, M., M. 1988, Ap. J. 326, 947. 